\title{
A death of a pregnant mother following Leptospirosis
}

Rathnaweera RHAI*

Department of Forensic Medicine, Faculty of Medicine, Karapitiya, Galle, Sri Lanka

*Corresponding author: Tel: 0094-772969060. E-mail address: ajithrathnaweera@yahoo.com

MLJSL. Vol 1. No 3. December. pp 20-22

\begin{abstract}
Introduction

Although leptospirosis is known to occur in Sri Lanka, its incidence has increased during the recent past. Because of the protean manifestations of leptospirosis it is sometimes misdiagnosed and underreported.
\end{abstract}

\section{Case report}

A 30 year old pregnant mother (POA 32 weeks) was transferred from a peripheral hospital with signs of icterus and vomiting for 3 days. She was treated by the Obstetrician as having HELLP syndrome. Haematological studies showed elevated liver enzymes and altered clotting profile. After an emergency caesarian section, she developed profuse haemorrhage and a subtotal hysterectomy was done. Although bleeding was arrested, her condition gradually deteriorated. She died in the ICU one week later.

\section{Autopsy findings:}

Yellow discolouration of conjunctiva and nail beds, and marked yellow stained pleural effusion and ascites were seen. Lungs were congested and heavy. Heart appeared flabby and yellow in colour. Kidneys were soft and enlarged with congested cortex. Cortico-medullary junction was well demarcated. Liver was enlarged and yellow.

Histopathology revealed extensive pulmonary haemorrhages and focal hepatic necrosis.

The serology report was positive for Leptospira (titer - 1600)

\section{Conclusions}

This death warrants the need of constant use of our knowledge in General Medicine, together with our specific knowledge which is confined to a particular specialty.

The serology report confirmed the autopsy diagnosis. Therefore, the need to carry out all the relevant specific investigations is highlighted here.

Key words: Leptospirosis, Pregnant mother, Pulmonary haemorrhage, Effusions

\section{Introduction}

Leptospirosis, also known as Weil's disease, an infectious disease, well known in all parts of the world since 1883, which was first identified by 
Lanka, its incidence has increased during the recent past. Because of the protean manifestations of leptospirosis it is sometimes misdiagnosed and under-reported.

Leptospirosis can manifest in many forms. Febrile illness with icterus, splenomegaly and nephritis (known as Weil's disease), acute febrile illness with severe muscle pain, febrile illness with pulmonary haemorrhages in the form of haemoptysis, jaundice with pulmonary haemorrhages, jaundice with heamaturea, meningitis with haemorrhages including sub conjunctival haemorrhage or febrile illness with cardiac arrhythmias with or without haemorrhages are some of the syndromes [2].

\section{Case report}

A 30 year old pregnant mother (POA 32 weeks) was transferred from a peripheral hospital with signs of icterus and vomiting for 3 days. She was suspected to be having HELLP syndrome and was managed accordingly by the Obstetrician. Haematological studies showed elevated liver enzymes and altered clotting profile. Her blood urea and serum creatinine levels were rising all the time. Emergency LSCS was performed and live non asphyxiated baby was delivered. After the delivery, she had developed profuse post partum haemorrhage. Subtotal hysterectomy was done and the bleeding was arrested. She was given 8 pints of blood, 12 pints of fresh frozen plasma and 4 pints of platelets. Her liver and renal functions continued to deteriorate. Her platelet count kept coming down. Ultimately she died in the ICU one week later.

\section{Autopsy findings:}

Yellow discolouration of conjunctiva and nail beds, and marked yellow stained pleural effusion and ascites were seen. Lungs were congested and heavy. Heart appeared flabby and yellow. Kidneys were soft and enlarged with congested cortex. Cortico-medullary junction was well demarcated. Liver was enlarged and yellow.
Histopathology revealed extensive pulmonary haemorrhages and focal hepatic necrosis. Kidneys showed evidence of acute renal tubular necrosis. Heart appeared unremarkable.

The serology report (Leptospira microscopic agglutination test) was positive with a titer of 1600.

\section{Discussion}

Leptospirosis is an endemic disease in Sri Lanka. The temperate climate, seasonal rainfall, humidity and other ecological factors have contributed to the maintenance of the disease in the country.

Majority of leptospiral infections are either sub clinical or result in very mild illness and recover without any complications [3]. However, a small proportion develops various complications due to involvement of multiple organ systems. In such patients, the clinical presentation depends upon the predominant organs involved and the case fatality ratio could be about $40 \%$ or more [3].

In most instances, leptospirosis presents as a sporadic disease especially among occupational groups like conservancy and agricultural workers. Such patients usually manifest as acute febrile illness with varying degrees of jaundice and renal dysfunction [4].

In this case, patient presented with vomiting and yellow discolouration of eyes with a history of low grade fever. The muddy contact history was not detected during the clinical management. As the main focus was on pregnancy and the probable diagnosis was HELLP syndrome, the other differential diagnoses were not adequately investigated.

But at the post-mortem examination, a through history was recorded and the muddy contact was detected. The general and macroscopic post-mortem appearances including yellow discolouration of conjunctiva and nail beds, marked yellow stained pleural effusion and ascites together with congested lungs and flabby heart pointed towards the possibility of 
leptospirosis. Therefore a blood sample was sent to serology and it was found to be positive.

\section{Conclusions}

This case warrants the need of constant use of our knowledge in General Medicine, together with our specific knowledge which is confined to a particular specialty.

The serology report confirmed the autopsy diagnosis. Therefore, the need to carry out all the relevant specific investigations is signified here. It can be agreed that this case highlights the necessity for the open and broad mindedness in medico-legal work.

\section{References}

1. Ruwanpura R, Rathnaweera A et al. Severe Pulmonary Leptospirois Associated With High Fatality Rate: An Autopsy Series in Galle, Southern Sri Lanka. Med J Malaysia Vol 67 No 6 December 2012

2. Kumar P, Clark M. Clinical Medicine: A textbook for medical students and doctors. 4th ed. London: W.B. Saunders,1998:45-46

3. Vijayachari $P$, Sugunan A, Shriram A. Leptospirosis: An emerging global public health problem. Indian Academy of Sciences 2008;

4. Ramalingaswamy V. Leptospirosis-/an introduction. In:Singhai RL, Sood OP, editors, Leptospirosis. Round table Conference series. New Delhi: Ranbaxy Science foundation. 1998. P.5-6 